\title{
How to choose a general practice computing system: comparison of commercial packages
}

\author{
Angela Daniels, Angela Coulter
}

General practitioners who consider computerising their patients' records are faced with a bewildering array of software systems from which to choose. The availability of independent advice and guidance on computer systems for general practitioners varies from region to region but on the whole is fairly poor. The Oxford community health project has developed experience in providing advice and training for general practitioners in the Oxford region on buying and using microcomputer systems in their practices. We describe how we obtained information on various systems and the facilities available in them.

The Oxford community health project has been a pioneer in promoting computing in general practice since it was established in 1970 as a collaborative venture between Oxford University's unit of clinical epidemiology and Oxford Regional Health Authority. The project was originally established to provide support to about 40 practices using the regional computer unit's mainframe and to undertake collaborative research in general practice. The mainframe service, which is still used by 23 practices, provides facilities for registering patients, recall, and listing records of patients with chronic diseases or other problems. ${ }^{1}$

Over the past five years several practices participating in the project have chosen the added advantages of on line computer time-for example, five practices shared an Alpha Micro system running British Medical Data Systems software, ${ }^{23}$ and others bought their own systems. In response to the growing demand for advice on microcomputing in the Oxford region the project has widened its scope to provide advice on the facilities of microcomputers and information systems in primary care. A priority was to develop a source of information on the systems available for general practitioners.

The first step in compiling the list of specialist systems and the facilities that they offered was to search reports on computing in general practice. By this means we identified 41 systems marketed by 40 suppliers that were or had been on the market in the past five years. All firms were contacted by letter and asked to provide information on their systems to help in the task of advising general practitioners. From the information received we constructed a chart to show the facilities each claimed to provide. We returned a copy of our interpretation of the system to each supplier with a request for comments, amendments, and corrections as well as details of any planned changes. We asked the suppliers to provide prices for two systems: a single user system with one printer and a 20 megabyte hard disk, and a multiuser system with four screens and four printers and a $\mathbf{4 0}$ megabyte hard disk. We also asked how many systems they had installed and in which areas; and whether they could install and support systems in the Oxford region.

\section{The systems}

We received replies from 17 suppliers. Fourteen of these were suppliers of current systems; the three others did not meet the criteria for inclusion in this report (combined hardware and software packages available throughout the United Kingdom). Five systems were no longer marketed, although they may still be in use in practices. Four letters were returned by the Post Office marked "not known at this address," and the remaining 15 companies did not reply.

All systems have a database that can hold such information as each patient's name, address, and title, date of birth, age, and sex. Once this information is recorded it is easy to institute call and recall systems and to compile registers of particular groups of patients. In addition, some systems offer the facility to record details of consultations, risk factors, social and medical problems, and diagnoses. Many systems can be used for prescribing and dispensing and for administrative purposes such as making appointments, doing accounts, and word processing. Some offer the additional facilities of audit and analysis of the practice activities. All of the systems are protected by passwords to ensure security of the data. Some of the main functions available in the different systems are described below. The table gives a summary of the information that we collected.

\section{INFORMATION ON PATIENTS}

Criteria for selecting patients-These indicate that various specific items of data can be used to select a patient's records from the practice register. The ability to select by date of birth, address, or partial address can be useful-for instance, selecting by street or postal code can identify patients living in a particular area, which can facilitate the planning of house calls, and selecting by individual address can identify residents of that address who have different surnames.

Recall-All systems investigated have call and recall facilities for various screening and immunisation procedures. Some have the recall intervals stated in the statement of fees and allowances (the "red book") programmed in to facilitate accurate claims for item of service fees.

Problem dictionary-Various classification systems exist, which are intended to cover almost any medical or social problem encountered in general practice. Standard problem dictionaries include the Royal College of General Practitioners' classification, ${ }^{4}$ the international classification of health problems in primary care ${ }^{5}$ the Read clinical classification, ${ }^{6}$ and the Oxford Medical Information System (OXMIS) classification.? In some systems users enter codes or abbreviations of their choice to define any items of interest to the practice. Some microcomputer systems can support any of the standard classification codes. Correspondence to: Angela
Coulter. 


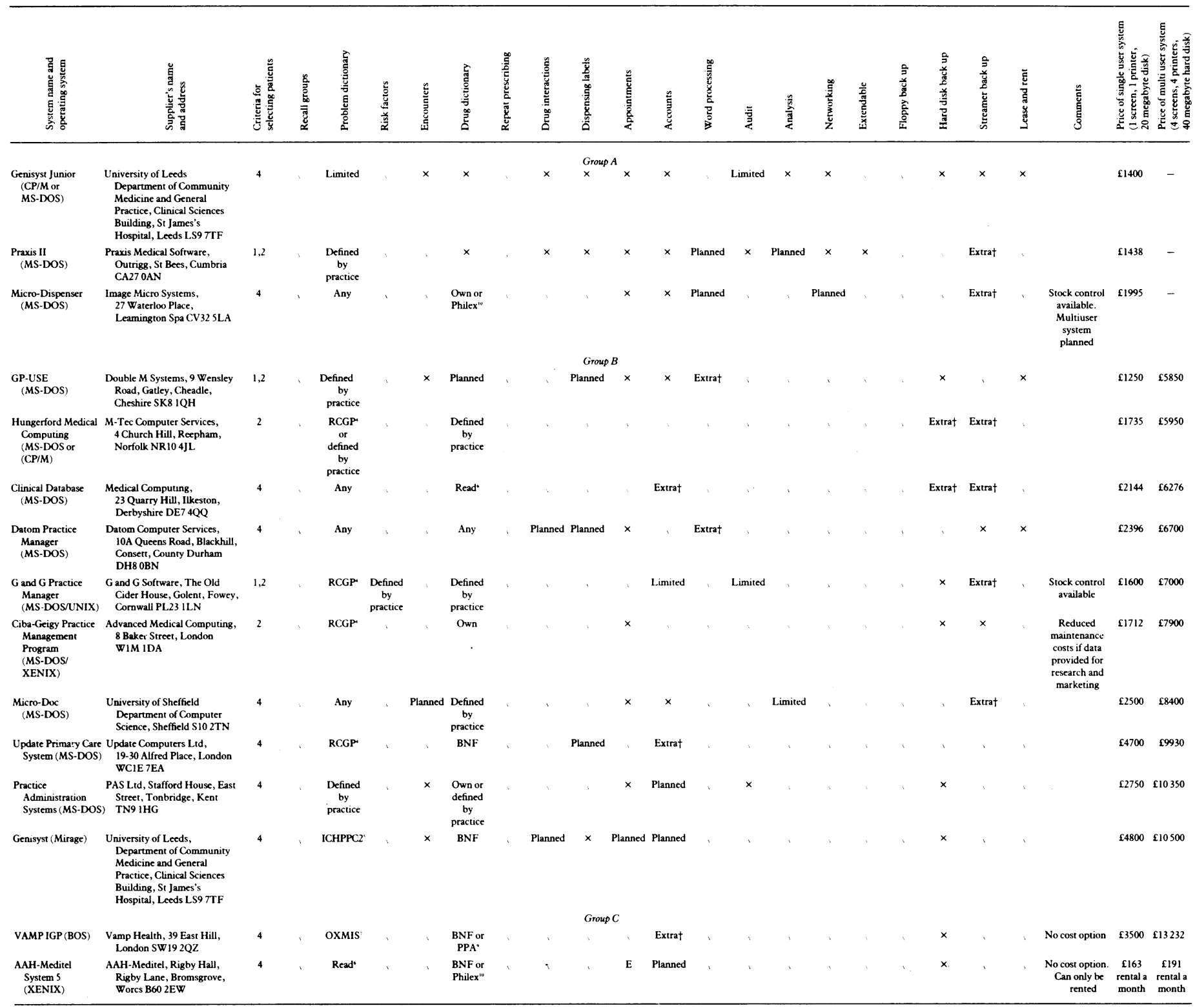

RCGP $=$ Royal College of General Practitioners. BNF $=$ British Narional Formulary. ICHPPC2 $=$ International classification of health problems in primary care. PPA $=$ Prescription Pricing Authority.

Risk factors - These enable doctors to record items such as blood pressure, weight, and whether the patient smokes and thereby facilitate health education.

Encounters-This implies that the system is capable of recording information about consultations and their outcome, including whether the consultation was in the surgery, at home, or by telephone and whether it resulted in a referral or follow up appointment.

\section{PRESCRIBING}

Drug dictionary-Some systems use standard drug dictionaries-for example, the British National Formulary, ${ }^{8}$ that developed by the Prescription Pricing Authority, ${ }^{9}$ Philex, ${ }^{10}$ or the Read codes, ${ }^{6}$ - while other systems use their own drug dictionaries or offer the facility for a practice to develop its own.

Repeats - This indicates that the system can handle repeat prescriptions. Some systems have standard drug doses and strengths built in, and this can save time as it removes the need to check them elsewhere.

Drug interactions-This indicates whether drug interactions and contraindications are checked each time a drug is prescribed.

Dispensing labels and stock control-Although most systems can produce medicine labels for dispensing, as far as we know only two of the systems can be used for integrated stock control.

\section{PRACTICE ADMINISTRATION}

Appointments-Some systems offer an integrated appointments system. This might include prompts for any outstanding preventive procedure due for patients about to be seen in the surgery. This can be a useful aid in opportunistic screening if there is no computer terminal on the doctor's desk.

Accounts-This may cover anything from calculating staff salaries to the ability to record all income and expenditure and to produce various reports and claims to the family practitioner committee.

Word processor-An integrated word processor allows personalised letters to be sent to any number of patients. Most systems come supplied with standard recall and immunisation letters, and in most systems these letters can be amended and new ones composed.

\section{PRACTICE INFORMATION}

Audit-This is the ability to compare various practice activities such as immunisation rates or prescribing patterns, either between partners or between years. 
Analysis-This can vary from simple statistical searches to producing sophisticated graphs and tables. The ability to produce age and sex counts of the practice population is particularly useful.

\section{SYSTEM INFORMATION}

Networking-This indicates whether a system can run several terminals and printers.

Extendable-An extendable system is one to which extra memory can be added.

Back up-Facilities for storing secure copies of the practice's database vary according to the system. The basic requirement is the extraction of an accurate copy of the data that can be stored elsewhere. For large practices where back up on to floppy disks may be time consuming facilities may exist for the data to be copied quickly on to magnetic tape on a tape streamer.

Lease and rental-Many suppliers now offer leasing or rental arrangements for their systems, which helps to spread the capital cost over several years.

Prices - The prices quoted in the table are those current at May 1988. The three systems in group A are single user systems currently available for less than $£ 2000$ for the hardware and software. Group B comprises 10 systems that offer multiuser facilities. The prices quoted, which range from under $£ 6000$ to over $£ 10000$ for a four screen and four printer system, will vary with the number of screens or printers required. Group $C$ comprises two systems that are available at little or no cost in return for data for research and marketing purposes. These systems are also available for purchase or rental. The Ciba-Geigy system in group $B$ offers reduced maintenance costs for practices that contribute anonymous marketing and research information. Most of the companies will also supply a single user version if required, and some can offer additional facilities at extra cost.

\section{How to choose a system}

Although many systems seem to offer similar facilities, the sophistication of each facility should be carefully investigated as well as its ease of use and capacity for expansion. The systems vary in the amount of data that they can store and the ways in which the data can be extracted, in report form, histograms, and graphs or as an audit of practice activities.

In addition to assessing the facilities the various computing systems can offer each practice needs to define carefully its present and predicted needs. ${ }^{112}$ In our experience many practices underestimate the amount of hardware (screens, printers, and disk storage capacity) that they will need, and so before buying any system a practice should ascertain the ease and cost of expanding that system when the demand arises.

A small practice wanting to use the computer for only registration and recall could consider one of the fairly cheap single user systems (group A or a single user version in group B). If, however, the practice is also intending to use the system for repeat prescriptions we suggest that a second printer dedicated to prescribing will be required. In this case it is important to check that the printer's memory is large enough to allow access to the screen for other purposes while the prescriptions are being printed.
Most practices find that they need more than one screen and printer, especially if they intend to use the system for a range of purposes. In this case the choice should be made from the multiuser systems in group B according to the facilities required and price limit. As requirements vary so much it is impossible to recommend a "best buy". that will meet all needs.

A practice intending to do extensive recording and research would do well to consider one of the "no cost" options (group C). These are worth considering if all members of the practice are committed to complying with the fairly stringent requirements for collecting data. Experience in Oxford practices has shown that even the best intentions can result in incomplete recording ${ }^{13}$ and the practices most likely to succeed are probably those in which every member has easy access to a computer terminal and the computer is used during consultations.

The "Micros for GPs" scheme ${ }^{14}$ prompted the British Medical Association to suggest in 1986 that regional advisers in computing should be appointed, but only six advisers have been appointed so far. The primary health care specialist group of the British Computer Society recently instigated a voluntary network of regional coordinators to provide advice and support. In the Oxford region response to training courses and meetings for doctors using computers has been enthusiastic. More advice and education are needed on a regional basis. This could be provided by the formation of local groups of users and by more collaboration between areas where advisers are already in post and those where these basic facilities are lacking.

New systems are being marketed all the time and companies are adding facilities to their current range. Every effort will be made to maintain the information in the table as an up to date overview of systems on the market. We would be pleased to hear from any suppliers whom we have not contacted or who failed to reply to our initial letter so that we may maintain and improve our advice service for general practitioners.

1 Tulloch A. The Oxford community health project. Oxford University Gazette 1981;42:20-2.

2 Stewart T, Mortlock M. Screening and recall using a microcomputer. Computer Update 1984;3:21-5.

3 Hayward JM, Appleby S. The true cost and profit of computing. Practice Computing 1986;4:6-9.

4 Royal College of General Practitioners. The classification and analysis of general practice data. London: RCGP, 1986.

5 Classification Committee of World Organisation of National Colleges, Academics and Academic Associations of General Practitioners and Family Academics and Academic Associations of General Practitioners and Family
Physicians. International classification of health problems in primary care. Physicians. International classification of

Oxford: Oxford University Press, 1979.
Read J, Benson TRR. Comprehensive coding. If Healthcare Computing Read J, Benson

Perry J, ed. OXMIS problem codes. Oxford: Oxford community health project, 1978.

8 British Medical Association and Pharmaceutical Society of Great Britain. British national formulary. No 15. London: BMA and Pharmaceutical Press, 1988

9 Prescription Pricing Authority. Drug master index. London: Department of Health and Social Security, 1986.

10 Anonymous. A new drug database for the GP computer. [Editorial.] The Physician November 1987;606.

11 Ritchie LD. Computers in primary care. London: Heinemann, 1984.

12 Fitter MJ, Garber JR, Herzmark GA, et al. A prescription for change. London: itter MJ, Garber

13 Mant D, Tulloch A. Completeness of chronic disease registration in general practice. Br Med f 1987;294:223-4.

14 Project Evaluation Group. General practice computing-evaluation of the "Micros for GPs" scheme. London: HMSO, 1985.

(Accepted 7 fune 1988) 